\title{
Feature Extraction and Classification of Surface EMG Signals for Robotic Hand Simulation
}

\author{
Sakshi Sharma \\ A.P. (ECE Department) \\ Chandigarh Engg. College \\ Landran, Mohali
}

\author{
Hemu Farooq \\ M.Tech Student (ECE Dept.) \\ Chandigarh Engg. College \\ Landran, Mohali
}

\author{
Nidhi Chahal \\ A.P. (ECE Department) \\ Chandigarh Engg. College \\ Landran, Mohali
}

\begin{abstract}
Feature Extraction and Classification of Surface Electromyography (EMG) signals provide an access for the development of Robotic Hand. EMG signals stands for electromyography signals. These are called the bio signals. Bio signal means a collective electrical signal acquired from any organ that represents a physical variable of interest. The EMG signal being a biomedical signal that measures electrical currents generated in muscles during its contraction representing neuromuscular activities. [1]. The nervous system always controls the muscle activity (contraction/relaxation). Hence, the EMG signal is a complicated signal, which is controlled by the nervous system and is dependent on the anatomical and physiological properties of muscles.

In this paper, we have discussed different steps in analyzing the EMG signals. The first step is to analyze the surface EMG signal from the subject's forearm using Discrete Wavelet Transform and extract features using the singular value decomposition.
\end{abstract}

The second step is to call the different feature values into linguistic terms by using Fuzzy Logic Classifiers in order to recognize different degrees of freedom like open to close, close to open etc.

This paper will give in depth insight in the field of EMG signal and has provided more efficient work when compared to conventional works and efficiency is $99 \%$.

\section{Keywords}

EMG signal, DWT, fuzzy classifier, feature extraction

\section{INTRODUCTION}

The Human Hand is versatile in its interaction. Imitating the versatilities and human dexterity in robotic design is a huge challenge, and requires a great depth of understanding of the human upper limb physiology and its robotic equivalent. Extensive research is being carried out across the globe in order to develop a human hand like prosthetic which can provide natural haptic functionality. Around the world there are large number of amputations due to diseases, accidents and war. There are many difficulties in their daily life, so it needs to design prosthetic control device which provides movement.

Electromyography technique is used for controlling such prosthetic hand. It represents the recorded electrical activities produced by skeletal muscles. The electrical activity generated during the contraction of skeletal muscles are called as EMG signals or Electromyography signals. These signals are called the biomedical signals as they measure the muscle response representing the neuromuscular activities [2]. EMG is applied to the study of skeletal muscle. The contraction of skeletal muscle is initiated by impulses in the neurons to the muscles and is usually under voluntary control. Skeletal muscle fibers are well supplied with neurons for its contraction. This particular type of neuron is called a" motor neuron" and it approaches close to muscle tissue but is not actually connected to it. One motor neuron usually supplies stimulation to muscle fibers [3].

Surface Electromyography signals (SEMG) is a type of bioelectrical phenomenon as the activity of muscle is associated on the surface of skin. Skin surface contains an abundance information regarding the muscle movement. SEMG can records biological signal generated on the skin surface during the muscle movement with the help of electrodes, so that these signals can be used to control the prosthetic or robotic hand [4].

\section{EMG SIGNALS}

EMG signals are the electrical responses generated during the contraction of a skeletal muscle. It measures muscle response or electrical activity in response to nerve's stimulation of the muscle. During the measurement, one or more small needles( or called invasive electrodes) are inserted through the skin into the muscle. The electrical activity picked up by the electrodes is then displayed on an oscilloscope (a monitor that displays electrical activity in the form of waves). EMG signals can also be taken by noninvasive electrodes. EMG measured by noninvasive electrodes is called surface EMG signals [5]. A pictorial view of EMG signals is shown in figure 1.

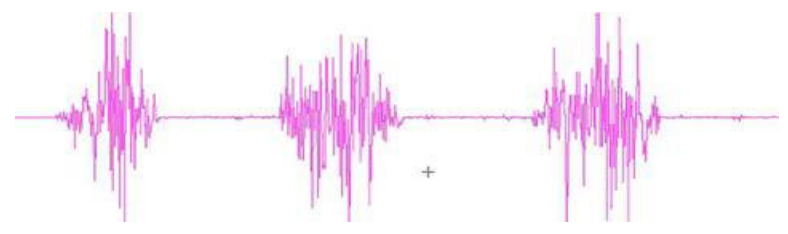

Fig 1: EMG Signal

After an electrode has been inserted, patient may be asked to contract the muscle, for example, by lifting or bending his hand. The action potential (size and shape of the signal) that this creates on the oscilloscope provides information about the stability of the muscle to respond when the nerves are stimulated. As the muscle is contracted more forcefully, more and more muscle fibers are activated producing action potentials. 


\section{USE OF EMG SIGNALS}

EMG signals have been used in such application as controlling active prosthesis, wheelchairs, exoskeleton robots, rehabilitation, silent speech recognition, and controlling video games as it can be measured on a human skin surface with noninvasive electrodes [6]. In commercially available prosthetic devices EMG signals have been exploited for a proportional control strategy. To improve its usability, a control strategy based on the classification of EMG signals has been widely studied, in such a strategy, a classifier is constructed for the surface EMG signals to recognize the intended human movements using classified movements to generate the corresponding behavior of the device. The EMG signal is also used to help to detect neuromuscular abnormalities [7].

\subsection{Need of Prosthetic Devices}

The quantity of amputees worldwide is assessed to be around 650 million and almost $10 \%$ of the amputee populace dwells in India. The expense of a customary fake appendage which is rural, non-adaptable and gives no common appendage attributes is around $\$ 40$ though; a totally robotized and very propelled appendage which practically imitate human appendage usefulness costs around $\$ 25,000-40,000$ [4]. There is an intense requirement for an ease, mechanized simulated appendage.

\section{HOW TO USE EMG SIGNALS}

The EMG signals cannot be used in the raw form. To use these signals first of all signal conditioning is done to reduce noise and to make more signal strength. After this there are some stages which should be followed by all control systems that are based on EMG signals. These are:-

\subsection{Data Acquisition and Data Segmentation}

In this stage, signal is acquired from body and is filtered to reduce noise produced by other electrical activities of body.

\section{A. Features Extraction and Dimensionality Reduction}

This stage converts raw signal obtained from stage 1 into feature vector. The feature vector represents relevant structure in the raw data. Dimensionality reduction eliminates redundant information in the feature vector, generating reduced feature vector.

\section{B. Feature Classification}

It involves pattern recognition. Since a classification algorithm is applied to reduced feature vector in order to obtain categories.

\section{Controller}

This stage translates categories to control commands for execution.

\section{FEATURE EXTRACTION}

There are three types of features in EMG signal based control system. These are time domain, frequency domain, timefrequency domain features. Here time-frequency domain features are to be considered. Mostly widely used wavelet transform method is used.

\section{B.i) Wavelet transform}

It is a transform where a signal is integrated with a shifted and scaled mother wavelet function [8]. The continuous wavelet function can be represented as: $w_{x}(a, b)=\int x(t)\left(\frac{1}{\sqrt{a}}\right) \psi^{x}\left(\frac{t-b}{a}\right) \mathrm{dt}$

Where $\mathrm{x}(\mathrm{t})$ represents the input signal, $\psi^{x}$ represents the complex conjugate of mother wavelet function and $\left(\frac{t-b}{a}\right)$ is the shifted and scaled version of wavelet having time $b$ and scale a.

\section{B.ii) Wavelet transform}

It is one of the most popular time-frequency representation techniques capable of representing a signal into twodimensional function of time and frequency. It offers several advantages in comparison to other frequency transform such as Fourier Transform and Cosine Transform. As, in another technique called Short Time Frequency Transform (STFT), a constant resolution is used at all frequencies but the DWT being a multi-resolution technique offers localization both in time and frequency. Thus the DWT exhibits good frequency resolution at low frequencies and good time resolution at high frequencies [9]. Hence, DWT is chosen to extract features from surface EMG signal.

The DWT coefficients of a signal $\mathrm{x}(\mathrm{n})$ may be obtained as:

$c(a, b)=\sum_{n=z} x[n] \psi_{a, b}[\mathrm{n}]$

$\psi_{a, b}[\mathrm{n}]=\frac{1}{\sqrt{a}} \psi\left(\frac{n-b}{n}\right)$

Where $\mathrm{a}$ is scale, $\mathrm{b}$ is translation and $\psi(\mathrm{n})$ represents discrete wavelet.

The DWT operation can be viewed by passing the signal $\mathrm{x}[\mathrm{n}]$ through two complementary filters that is low pass filter having impulse response $\mathrm{g}[\mathrm{n}]$ and high pass filter having impulse response $\mathrm{h}[\mathrm{n}]$ and may be represented as:

$y_{g}[\mathrm{n}]=\sum_{k=-\infty}^{\infty} x[k] g[2 n-k]$

$y_{h}[\mathrm{n}]=\sum_{k=-\infty}^{\infty} x[k] h[2 n-k]$

Thus DWT helps in analyzing the signal at different frequency band with different resolution.

\section{Feature classification}

The output of feature extraction must be converted into linguistic terms which can be understood. So, it is necessary to classify the features. There are various methods involving the classification of features [10].

\section{C.i)Fuzzy logic classifier}

The fuzzy logic was introduced by in 1965 by Lotfi Zadeh. It is a mathematical tool for dealing with uncertainty, imprecision and information granularity. Fuzzy theory employs fuzzy sets, membership functions, rules, fuzzification, defuzzification. Fuzzy sets model the uncertainty associated with vagueness, imprecision and lack of information regarding the problem, involving the linguistic variables such as low, medium, high, often, few etc. 


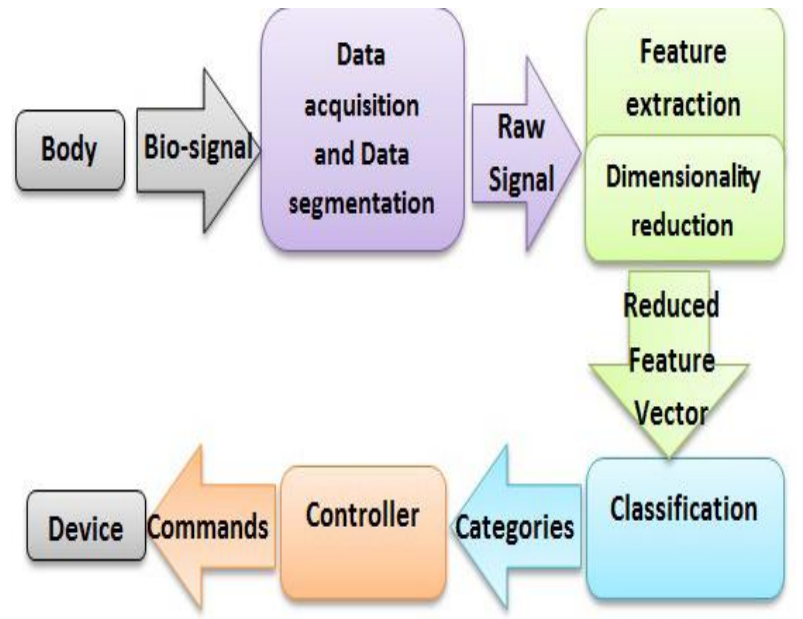

Fig1. Shows the block diagram representation of the proposed research

\section{RESULT AND DISCUSSIONS}

Initially the EMG Signal is taken from the readings acquired from different persons. Now this signal is processed using tools DWT and Fuzzy Logic Classifiers so that the signal is in its purest form or understable in terms of linguistic forms. Different robotic hand movement parameters like ON/OFF time, maximum and average voltage are calculated. The highest accuracy that has been calculated is $99 \%$.

\section{EMG Based Robotic Arm: Open Close Action}

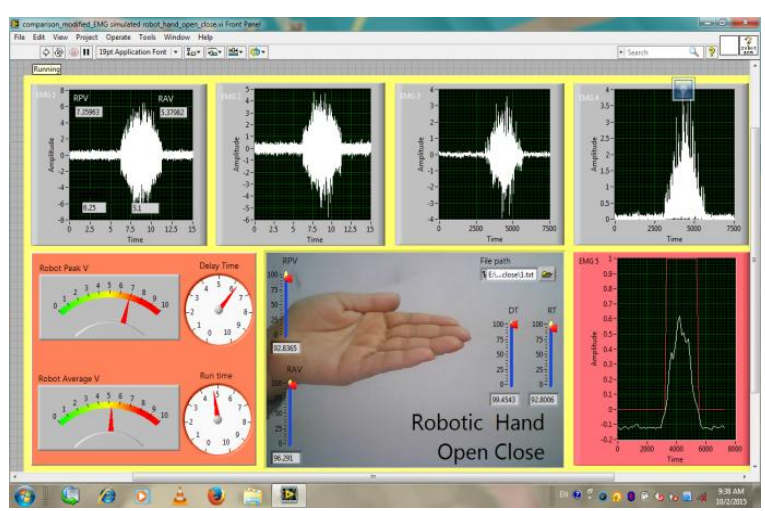

Fig.2 shows the open close movement of $1^{\text {st }}$ person.

The accuracy in terms of delay time, run time, robot average voltage, robot peak voltage is shown above. Accurate Delay Time calculated is $99.4543 \%$. It is the time spent from standstill condition to starting of motion.

Accurate Run Time calculated is $92.8006 \%$. It is the total time spent during the complete one motion of hand (open close).

Accurate Robot Average Voltage calculated is $96.291 \%$. It is the average force applied to complete any hand movement. Accurate Robot Peak Voltage calculated is $92.8365 \%$. It is the peak force that is maximum force applied to complete any hand movement. This is applied to 5 persons and the results are obtained as below:

\subsection{Results of the Proposed Work}

Table 1: Maximum Accuracy Obtained for Delay Time

\begin{tabular}{|l|l|}
\hline Delay Time & DT Accuracy $(\%)$ \\
\hline $1^{\text {st }}$ Person & 99.4 \\
\hline $2^{\text {nd }}$ Person & 94.9 \\
\hline $3^{\text {rd }}$ Person & 94 \\
\hline $4^{\text {th }}$ Person & 94.9 \\
\hline $5^{\text {th }}$ Person & 97.2 \\
\hline
\end{tabular}

Table 2: Maximum Accuracy Obtained for Run Time

\begin{tabular}{|l|l|}
\hline Run Time & RT Accuracy (\%) \\
\hline $1^{\text {st }}$ Person & 92.8 \\
\hline $2^{\text {nd }}$ Person & 97.4 \\
\hline $3^{\text {rd }}$ Person & 99.4 \\
\hline $4^{\text {th }}$ Person & 97.4 \\
\hline $5^{\text {th }}$ Person & 94.6 \\
\hline
\end{tabular}

Table 3: Maximum Accuracy Obtained for Robot Average Voltage

\begin{tabular}{|l|l|}
\hline Robot Average Voltage & RAV Accuracy $(\%)$ \\
\hline $1^{\text {st }}$ Person & 96.3 \\
\hline $2^{\text {nd }}$ Person & 65.3 \\
\hline $3^{\text {rd }}$ Person & 91.7 \\
\hline $4^{\text {th }}$ Person & 98.2 \\
\hline $5^{\text {th }}$ Person & 99.6 \\
\hline
\end{tabular}

Table 4: Maximum Accuracy Obtained for Robot Peak Voltage

\begin{tabular}{|l|l|}
\hline Robot Peak Voltage & RPV Accuracy $(\%)$ \\
\hline $1^{\text {st }}$ Person & 92.8 \\
\hline $2^{\text {nd }}$ Person & 84.3 \\
\hline $3^{\text {rd }}$ Person & 96 \\
\hline $4^{\text {th }}$ Person & 91.1 \\
\hline $5^{\text {th }}$ Person & 95.9 \\
\hline
\end{tabular}




\subsection{Graphical Comparison between the Proposed Work and the Conventional Works}

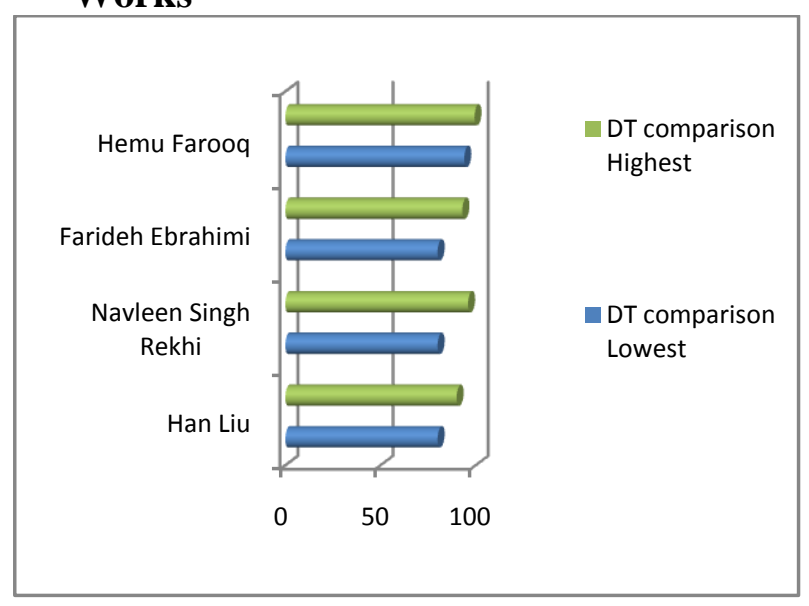

Fig.3 Bar Graph Comparison of Proposed system and Previous Systems in terms of DT

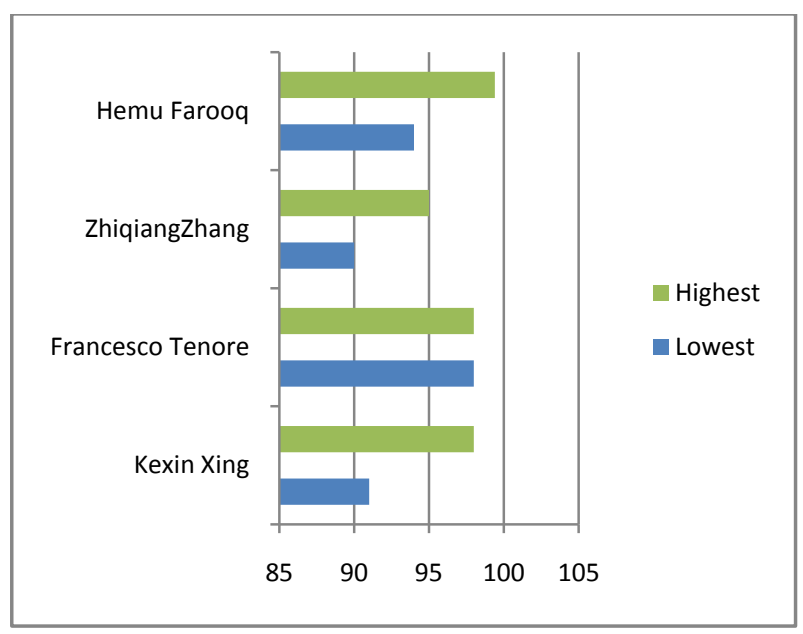

Fig.4 Bar Graph Comparison of Proposed System and Previous Systems in terms of RT

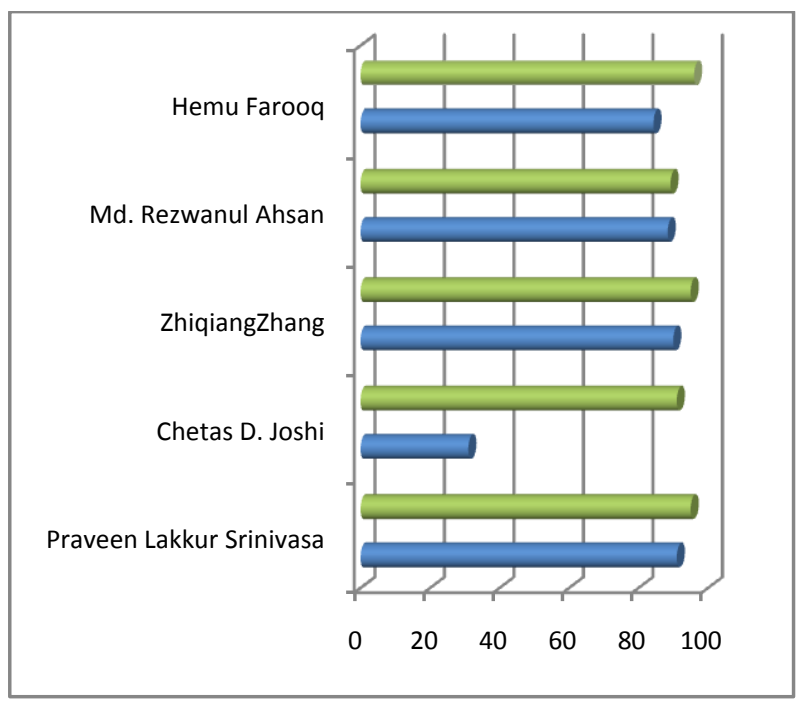

Fig.5 Bar Graph Comparison of Proposed System and Previous Systems in terms of RPV
7. CONCLUSION AND FUTURE SCOPE

Around the world, there are large number of hand amputations due to diseases, accidents, various health ailments etc. So the work done in this direction will be a great boon for disabled and handicapped people. It is very tough to automate the artificial hand from EMG signal received. This technology is in its initial stage and lot of work has to be done in this field. Different softwares and algorithms has been developed and used to overcome the problem. But due to high cost of the artificial hands, it is not used by the normal human being. Therefore it is very important to reduce the cost and at the same time improve the technology.

The EMG signal received is very complex and raw in nature. The proposed work utilizes this EMG signal acquired from body and converts it into useful form. The EMG in this form has important information so it is processed using tools like DWT and Fuzzy Logic Classifiers.

The work has a great scope when accomplished using software, microcontrollers etc. It can also be done in software simulation when burnt in microcontroller to get the work done. The is really great field to do some work in the field of humanities and technology also because it is really great work to give working artificial hand to some disabled persons.

\section{SECTIONS}

The first section gives the introduction about Human hand and surface EMG signals. Section 2 and section 3 introduces about the EMG signals and use of EMG signals. Section 4 details us about how to use EMG signals. Section 5 gives the results of the Proposed research. Section 6 explains the conclusion and future scope of the research . Last section gives the list of references which are used for literature review.

\section{REFERENCES}

[1] Praveen Lakkur Srinivasa, Nagananda S. R,"Development of two Degree of freedom (DOF) Bionic Hand for Below Elbow Amputee," Proceedings of the IEEE international conference on Electronics, Computing and Communication Technologies, Bangalore, 17-19 Jan. 2013, pp.1-6

[2] Shaikh Anowarul Fattah, A. B. M. Sayeed Ud Doulah, Md. Asif Iqbal and Celia Shahnaz, Wei-Ping Zhuand M. Omair Ahmad, "Identification of Motor Neuron Disease Using Wavelet Domain Features Extracted from EMG Signal," Proceedings of the IEEE International Symposium on Circuits and systems, Beijing, 19-23 May 2013, pp.1308-1311

[3] Chetas D.Joshi, Uttama Lahiri and Nitish V. Thakor, "Classification of Gait Phases from Lower Limb EMG Application to Exoskeleton Orthosis," IEEE Transactions on point-of-Care Healthcare Technologies, Banglore, 1618 Jan 2013, pp.228-231

[4] Zhiqiang Zhang, Charence Wong, Guang-Zhong Yang, "Foorearm Functional movement Recognition Using Spare Channel Surface Electromyography," Proceedings of the IEEE International Conference on Body Sensor Networks, 6-9 May 2013, pp.1-6

[5] Kexin Xing, Qi Xu, YeguiLin, "Identification Scheme of Surface Electromyography of Upper Limb Movement," Journal of Networks, Vol. 8, No. 4, April 2013 
[6] Francesco Tenore, Ander Ramos,Amir Fahmy, Soumyadipta Acharya, Ralph Etienne-Cummings and Nitish V. Thakor, "Towards the control of individual Fingers of a Prosthetic Hand Using Surface EMG Signals," Proceeding of International Conference on Engineering in Medicine and Biology Society, Lyon August 23-26, 2007, pp.6145-6148

[7] Md. Rezwanul Ahsan, Muhammad Ibn Ibrahimy, Othman O. Khalifa, "Electromyography (EMG) Signal based Hand Gesture Recognition using Artificial Neural Network (ANN)," Proceedings of International Conference on Mechatronics (ICOM), Kaula Lumpur, Malaysia 17-19 May 2011, pp.1-6

[8] Han Liu, Yun-Wei Huang and DingLiu, "Multi-class Surface EMG classification using Support Vector Machines and Wavelet Transform," Proceedingsof the $8^{\text {th }}$ World Congress on Intelligent Control and Automation, Jinan, July 6-9 2010, pp.2936-2967

[9] Eldin Henry Shroffe D, P. Manimegalai, "Hand Gesture Recognition Based on EMG Signals Using ANN," International Journal of Computer Application Issue 3, Vol. 2, April 2013

[10] Navleen Singh Rekhi, Ajat Shatru Arora, Sukhwinder Singh, Dilbag Singh, "Multi-Class SVM Classification of Surface EMG Signal for Upper Limb Function," Proceedings of
[11] ICBBE 3rd International Conference on Bioinformatics Biomedical Engineering, Beijing, 11-13 June, 2009, pp.1-4

[12] Farideh Ebrahim, Mohammad Mikaeili, Edson Estrada, Homer Nazeran, “Automatic Sleep Classification Based on EEG Signals by using Neural Networks and Wavelet Packet Coefficients," Proceedings of $30^{\text {th }}$ Annual IEEE International Conference, August 20-24, 2008, pp.11511154

[13] A.Phinyomark, C. Limsakul, P. Phukpattaranont, "Application of Wavelet Analysis in EMG Feature Extraction for Pattern Classification," Measurement Science Review, Vol. 11, No. 2, 2011

[14] Zhiguo Yan, Zekun Liu, "The Study on Feature Selection Strategy in EMG Signal Recognition," Proceedings of 2013 ICME International Conference on Complex Medical Engineering, May 25-28, pp. 711-716

[15] Rajesh Kumar Tripathy, Ashutosh Acharya, Sumit Kumar Choudhary, Santosh Kumar Sahoo, "Artificial Neural Network based Body Posture Classification from EMG Signal Analysis”, Indonesiann Journal of Electrical Engineering and Informatics (IJEEJ), Vol. 1, No. 2 June 2013, pp. 59-63 